\title{
Respuesta fisiológica de especies arbóreas al anegamiento. Nuevo conocimiento sobre especies de interés en el arbolado urbano de Bogotá
}

\author{
Physiological responses of tree species to waterlogging condition. \\ New Knowledge about species of interest in the urban trees of Bogota
}

\section{Darwin Moreno Echeverry ${ }^{\circledR}$, Diana Carolina Useche Rodríguez ${ }^{2} \odot$ y Helber Enrique Balaguera ${ }^{3} \odot$}

Moreno-Echeverry, D., Useche-Rodríguez, D. y Balaguera, H. (2019). Respuesta fisiológica de especies arbóreas al anegamiento. Nuevo conocimiento sobre especies de interés en el arbolado urbano de Bogotá. Colombia Forestal, 22(1), 51-67.

DOI: http://dx.doi.org/10.14483/2256201X.13453

Recepción: 7 de junio de 2018

\section{Resumen}

Los efectos del calentamiento global en las zonas urbanas hacen preponderante el estudio de especies arbóreas en diferentes condiciones de estrés abiótico, como el anegamiento. En este estudio se analizó el comportamiento fisiológico de seis especies nativas con cuatro niveles de anegamiento, determinando el estado hídrico, el comportamiento fotosintético y la pérdida de electrolitos. De acuerdo a los parámetros evaluados, se recomienda establecer Quercus humboldtii y Ficus tequendamae en zonas con altas precipitaciones y con tendencia al anegamiento debido a la tolerancia que se evidenció. Esto permite que estas zonas en Bogotá mantengan el arbolado urbano bajo escenarios de cambio climático. La estabilidad de las membranas celulares y la eficiencia máxima fotosintética del fotosistema II pueden ser usados como marcadores de tolerancia a condiciones de anegamiento en árboles.

Palabras clave: adaptación, estrés en plantas, Fv/Fm, fotosíntesis, inundación, fuga de electrolitos, cambio climático.
Aprobación: 29 de octubre de 2018

\begin{abstract}
The global warming effects in urban areas make preponderant the study of tree species at different conditions of abiotic stress, such as waterlogging. Therefore, it is important to conduct research to select species tolerating these environmental conditions and to find physiological markers for these selection processes. In this study, we analyzed the physiological behavior of six native species with four levels of waterlogging, determining the hydric state, the photosynthetic behavior and loss of electrolytes. According to the parameters evaluated, it is recommended to establish Quercus humboldtii and Ficus. tequendamae in areas with high rainfall and with a tendency to waterlogging. This recommendation arises due to the evident tolerance, allowing these areas in Bogota to maintain urban trees under climate change scenarios. The stability of the cell membranes and the maximum photosynthetic efficiency of photosystem II can be used as markers for tolerance under waterlogging conditions in trees.

Key words: adaptation, stress in plants, Fv/Fm, photosynthesis, flood, electrolyte leakage, climate change.
\end{abstract}

1 Facultad de Ciencias Agrarias, Universidad Nacional de Colombia. Grupo de Investigación Horticultura. Bogotá, Colombia. dlmorenoe@unal.edu.co. Autor para correspondencia

2 Subdirección Científica, Jardín Botánico de Bogotá José Celestino Mutis. Grupo de Investigación y Conservación de la Flora de la Región Capital como Estrategia de Adaptación al Cambio Climático. Bogotá, Colombia.

3 Facultad de Ciencias Básicas. Universidad el Bosque, Bogotá Colombia; Grupo de investigaciones agrícolas, Universidad Pedagógica y Tecnológica de Colombia. Tunja, Colombia. 


\section{INTRODUCCIÓN}

El surgimiento del arbolado urbano ha sido preponderante en las ciudades, debido a su papel ornamental, a los múltiples servicios ecosistémicos que brinda a los ciudadanos y a su contribución en la adaptación al cambio climático (Jim y Zhang, 2013; Kirkpatrick, Davison y Daniels, 2013; Ordoñez, 2015). Sin embargo, las complejas condiciones ambientales que se presentan en las zonas urbanas (temperaturas elevadas, reducción de la humedad relativa y altos niveles de contaminación atmosférica), se consideran como los principales factores que condicionan la susceptibilidad del arbolado a otros factores como los bióticos (Sjöman y Nielsen, 2010; Swoczyna, Klaji, Pietkiewicz y Borowsky, 2015; McPherson, Berry y Svan, 2018). Se ha reportado que numerosas especies arbóreas nativas no tienen la capacidad de enfrentar los efectos causados por estrés de tipo ambiental, que se generan en las zonas urbanas (Cekstere, Nikodemus y Osvalde, 2008; Borowski y Pstragowska, 2010) y que se esperan intensificar debido al efecto del calentamiento global en una gran variedad de ecosistemas, incluyendo los urbanos (Wilby, 2008).

En diferentes investigaciones se ha reportado que una de las limitantes que presenta el arbolado urbano para establecerse y prosperar es el anegamiento: la escorrentía generada por las superficies pavimentadas, la topografía del terreno, las fuertes precipitaciones, el sistema de acueducto y alcantarillado y a los eventos climáticos extremos (Hundecha y Bardossy, 2004; Bailey-Serres y Voesenek, 2008; Salazar et al., 2012). Así mismo, el efecto negativo del anegamiento en los árboles se puede incrementar dependiendo de las condiciones edafológicas que presente el sitio de siembra (compactación, textura fina, profundidad efectiva limitada, mal drenaje) y por decisiones equivocadas con respecto al establecimiento (siembra de especies arbóreas en lugares inapropiados).

El estrés de las plantas por anegamiento se genera cuando el suelo está saturado con agua y, por lo tanto, el aire contenido en los poros es desplazado, generando una disminución de oxígeno $\left(\mathrm{O}_{2}\right)$ disponible para las raíces (Caudle y Maricle, 2014; Bhatt et al., 2015). Esto propicia condiciones de hipoxia (Dat et al., 2004) y, en consecuencia, una disminución en el metabolismo de la raíz causando daño al sistema radical de la planta. Lo anterior acompañado de la acumulación de fitotoxinas en el suelo como manganeso, hierro reducido, súlfidos y amonio, así como altas concentraciones de $\mathrm{CO}_{2}$ y etileno, causa senescencia prematura, clorosis y necrosis foliar, pérdida del rendimiento y hasta la muerte de la planta (Dell’Amico, TorreciIlas, Rodríguez, Morales y Sánchez-Blanco, 2001; Lucassen et al., 2002; Bailey-Serres y Voesenek, 2008). Una reducción del $\mathrm{O}_{2}$ en el suelo por anegamiento genera una disminución en la tasa de respiración y limita así la producción de adenosín trifosfato (ATP) (Pardos, 2010). Por consiguiente, la respiración aeróbica bajo esta condición se ve limitada ocasionando acumulación de piruvato, lactato y etanol (Colmer et al., 2006) y desencadena una serie de efectos fisiológicos y metabólicos en las plantas (Bailey-Serres y Voesenek, 2008).

La primera respuesta de las plantas a condiciones de anegamiento es el cierre de sus estomas, lo cual afecta los procesos de conductancia estomática y la asimilación de $\mathrm{CO}_{2}$ (King, Robinson y Cameron, 2012). Debido al cierre estomático, se reducen los procesos fotosintéticos en las plantas, tanto por la disminución de dióxido de carbono en el espacio intercelular, como por el desacople generado entre las dos fases de la fotosíntesis, aumentando la formación de especies reactivas de oxígeno y el daño a nivel de las membranas celulares y los fotosistemas. (King, Robinson y Cameron, 2012; Kissmann, Veiga, Eichemberg y Habermann, 2014). La disminución en el intercambio de gases acompañado de la limitación en la absorción de elementos esenciales como nitrógeno $(\mathrm{N})$, fósforo (P) y potasio $(\mathrm{K})$ como consecuencia de cambios en el $\mathrm{pH}$ y relaciones iónicas en el suelo bajo condiciones de anegamiento, afectan el crecimiento y desarrollo de la planta (Banach et al., 2009; 
Baracaldo et al., 2014; Moreno y Fischer, 2014; Nyman y Lindau 2016).

Sin embargo, las especies tolerantes tienen la capacidad de responder al anegamiento sobreviviendo y creciendo bajo esta condición, debido a diferentes factores que incluyen adaptaciones de tipo fisiológicas, morfológicas y anatómicas (Kreuzwieser et al., 2009). Entre los procesos más reportados en la literatura científica se encuentra la rápida disminución de la conductáncia estomática, aumentando el uso eficiente del agua (Du, Xu, Wu, Tu y Zheng, 2012), la formación de hojas epinásticas y la inducción de raíces adventicias (Aldana, García y Fischer, 2014; Yetisir, Caliskan, Soylu y Sakar, 2006) y la acumulación de osmolitos compatibles para realizar ajuste osmótico (Du et al., 2012; Voesenek y Serres, 2013).Otro mecanismo de aclimatación consiste en la rápida degradación de las clorofilas para evitar la absorción excesiva de energía lumínica y por ende el daño en el fotosistema II (PSII) (Du et al., 2012).

Las seis especies nativas arbóreas y arbustivas seleccionadas para la presente investigación se encuentran ampliamente distribuidas en la ciudad de Bogotá, representan el $6.4 \%$ del arbolado urbano (70 968 plantas). A la fecha se encuentran establecidos 3607 árboles de Ficus tequendamae, 15616 árboles de Quercus humboldtii, 7561 árboles de Junglas neotropica, 1624 arbustos de Dalea coerulea, 29423 arbustos de Dodonaea viscosa y 13137 arbustos de Senna viarum (Sigau, 2018).

Las especies seleccionadas tienen gran potencial en diferentes áreas. A nivel ambiental son usadas en repoblamiento forestal, restauración de tierras degradadas, protección de nacimientos, enriquecimiento de hábitat, corredores lineales, corredores multiestrato, corredores riparios, puntos de paso, controladores de la erosión y restauración ecológica (Tullah, Hussain y Ibrar, 2010; Paz, 2012; Pérez, Villalba y Almanza, 2013; Campoe et al., 2014; Rowshan, Farhadi y Najafian, 2014). En la economía tienen usos en la industria maderable, generación de productos medicinales, alimenticio (forraje), además de tener propiedades antimicrobianas, antimicóticas e insecticidas (Kamdem et al., 2012; Hussain et al., 2013; Mostafa et al., 2014; Ali et al., 2014).

En la actualidad existe poca información sobre la respuesta de estas especies a diferentes condiciones ambientales limitantes para su desarrollo (Glenz, Lougulescu, Kienast y Schalaepfer, 2008; Almeida, Pinto, Correia, Santos y Concalves, 2013; Hill, Guerin, Hill y Watling, 2014; Sepulveda, Diez, Moreno, León y Osorio, 2014; Corcobado, Cubera, Juárez, Moreno y Solla, 2014). No obstante, la información multidisciplinaria que se ha realizado entorno a estas especies, la generación de nuevo conocimiento, permitirá obtener información sobre la selección de árboles. Si se desea hacer una transición del arbolado urbano de la ciudad a una estructura forestal urbana más estable y resistente a los efectos del calentamiento global, este es un aspecto importante para los que toman las decisiones (Quintero y Jaramillo, 2012; Sghaier-Hammami et al., 2013; McPherson et al., 2018).

El objetivo de la investigación fue conocer la respuesta fisiológica a nivel del estado hídrico, el comportamiento fotosintético y la pérdida de electrolitos de seis especies arbóreas nativas de Colombia a diferentes periodos de anegamiento, para que sean usadas en el arbolado urbano de la ciudad de Bogotá e identificar marcadores fisiológicos que permitan seleccionar especies arbóreas tolerantes a dichas condiciones.

\section{MATERIALES Y MÉTODOS}

El ensayo se realizó en una parcela experimental bajo condiciones semicontroladas en el vivero La Florida $\left(7^{\circ} 43^{\prime} 34^{\prime \prime} \mathrm{N}\right.$ y $74^{\circ} 06^{\prime} 00^{\prime \prime} \mathrm{O}, 2640 \mathrm{~m}$ de altitud) del Jardín Botánico de Bogotá José Celestino Mutis, ubicado en la localidad de Engativá, Cundinamarca. Allí se registró una temperatura diurna promedio de $21.8^{\circ} \mathrm{C}$, humedad relativa promedio de $58 \%$ y temperatura de suelo promedio de $23.4^{\circ} \mathrm{C}$.

Se seleccionaron seis especies nativas, tres arbóreas (Quercus humboldtii, Juglans neotropica y 
Ficus tequendamae) y tres arbustivas (Dalea coerulea, Dodonaea viscosa y Senna viarum) proporcionadas por el vivero La Florida. Se utilizaron 140 plantas por especie, las cuales se encontraban en estado juvenil y en buenas condiciones fitosanitarias.

Las plantas de las seis especies se dejaron durante 20 días en proceso de aclimatación a las condiciones del semitecho y se generaron cuatro niveles de estrés por anegamiento: 7, 14, 21 y 28 días de anegamiento (dda). El anegamiento se generó en tres camas de $10 \mathrm{~m}$ de longitud, $1 \mathrm{~m}$ de ancho y $0.6 \mathrm{~m}$ de profundidad. Las camas fueron permeabilizadas con plástico negro y llenadas con agua potable. El nivel del agua siempre se mantuvo $10 \mathrm{~cm}$ por encima de la superficie del suelo de las plantas, que se ubicaron en las camas en sus respectivas bolsas de trasplante. El suelo contenido en las bolsas de trasplante tenía las mismas características físico-químicas en todos los tratamientos evaluados: presentaba una textura franco arcillosa y un contenido de carbono orgánico de $4.6 \%$. Los muestreos se realizaron de forma aleatoria en cuatro plantas por tratamiento, con diferencial número de hojas analizadas dependiendo del tipo de parámetro a medir.

\section{Estado hídrico de las plantas}

Para determinar el estado hídrico de las plantas se realizó mediciones del contenido relativo de agua (CRA), tomando tres muestras por planta de cuatro plantas por tratamiento. Las muestras fueron retiradas de las plantas y pesadas inmediatamente, lo cual se registró como el peso fresco (PF). Posteriormente, se colocaron en una cámara húmeda para que el tejido llegase a su capacidad máxima de hidratación y se tomó el peso de saturación 24 horas después (PSat). Luego de tomar este peso, la muestra se ubicó en una bolsa de papel y fue llevada al horno por 36 horas a $80^{\circ} \mathrm{C}$, donde posteriormente se tomó el peso seco (PS). Con los valores de los tres pesos anteriores se utilizó la siguiente fórmula para conocer el contenido relativo de agua de las plantas: $\mathrm{CRA}=(\mathrm{PF}-\mathrm{PS}) /(\mathrm{PS}$ at-PS) $\mathrm{x} 100$ (Liu, Shahanzari y Andersen, 2005).

\section{Respuesta fotosintética}

Las variables fisiológicas para determinar el intercambio gaseoso de las plantas fueron tasa fotosintética (A) y conductancia estomática (gs). Se utilizó una densidad de flujo fotónico diferencial para cada una de las especies evaluadas de acuerdo a los puntos de saturación generados en las curvas de luz. Se midió la fluorescencia de la clorofila a, determinando la eficiencia máxima del PSII (Fv/Fm), oscureciendo las hojas con bolsas de papel aluminio durante 60 minutos y realizando las mediciones bajo condiciones de oscuridad. Estas mediciones se realizaron en tres hojas por planta de cuatro plantas por tratamiento, en hojas desarrolladas y completamente expandidas del tercio medio. Tanto el intercambio gaseoso como la fluorescencia de la clorofila a fueron determinados con un IRGA GFS-3000 (Portable Gas Exchange-Fluorescence System).

\section{Pérdida de electrolitos}

Este parámetro se midió a partir de 10 discos con un diámetro de $2.5 \mathrm{~mm}$ de hojas del tercio medio de las plantas, que fueron lavadas con agua desionizada para remover los electrolitos adheridos a la superficie. Los discos de hoja se colocaron en tubos falcon con $4 \mathrm{ml}$ de agua desionizada a temperatura ambiente. La conductividad eléctrica (CE) fue determinada a las 22 horas usando un conductivímetro (HI 9835, HANNA instruments). Después de la medición de las 22 horas la muestra se calentó a $90^{\circ} \mathrm{C}$ por 15 minutos y se determinó la $\mathrm{CE}$, tomando este valor como la máxima pérdida de electrolitos. Los valores de CE presentados se expresaron como porcentaje respecto al mayor valor mediante la fórmula $\mathrm{PE}=(\mathrm{EC} 1 / \mathrm{EC} 2) \mathrm{X} 100$, donde $\mathrm{PE}=\%$ de pérdida de electrolitos; $\mathrm{EC} 1=$ pérdida de electrolitos a las 22 horas; $\mathrm{EC} 2=$ pérdida de electrolitos después de calentar a $80^{\circ} \mathrm{C}$ (Rodríguez et al., 2005). 


\section{Análisis estadístico}

Se realizaron pruebas de supuestos de normalidad (Shapiro-Wilk) y de homogeneidad de varianzas. Así mismo, la prueba de comparación múltiple de Tukey con un nivel de confianza del 95\% (P $\leq$ $0.05)$ con el software estadístico Statistical Analysis System $\left(\mathrm{SAS}^{\circledR}\right)$ versión 9.2.

\section{RESULTADOS}

El contenido relativo de agua (CRA) varió diferencialmente de acuerdo a la especie y al nivel de estrés. Para Q. humboldtii (figura 1a), el CRA disminuyó ligera pero significativamente desde los 7 dda hasta los 21 dda, presentó una disminución significativamente mayor a los 28 dda y alcanzó un CRA del $71 \%$ al momento de mayor estrés. $F$. tequendamae (figura 1c) presentó un comportamiento similar a la especie anterior, disminuyendo su CRA ligeramente y solo con diferencias significativas hasta los 21 y 28 dda, alcanzando valores del $73 \%$ a los 28 dda. J. neotropica (figura 1 b) disminuyó drásticamente el CRA, alcanzando valores del $22 \%$ a los 21 y $28 \mathrm{dda}$, con diferencias significativas en su disminución entre los 0 y los 21 dda. Este parámetro presentó una disminución en $D$. coerulea (figura $1 \mathrm{~d}$ ) a medida que el nivel de estrés era mayor, presentando valores del $22 \%$ a los 28 dda. Para D. viscosa (figura 1e), el CRA se vio ligeramente disminuido sin diferencias estadísticas a los 7 dda y presentando un valor del $60 \%$ al momento de mayor estrés. El CRA presentó una gran disminución en $S$. viarum (figura 1f) con diferencias significativas entre todos los momentos de estrés con respecto al control, llegó a valores cercanos al $20 \%$ a los 28 dda.

\section{Intercambio gaseoso}

La fotosíntesis (A) presentó una ligera disminución para Q. humboldtii (figura 2a) variando desde 17 umoles $\mathrm{CO}_{2} \mathrm{~m}^{-2} \mathrm{~s}^{-1}$ hasta $14 \mu$ moles $\mathrm{CO}_{2} \mathrm{~m}^{-2} \mathrm{~s}^{-1}$, al momento de mayor estrés, con diferencias significativas. Para J. neotropica (figura 2b) se observó una disminución en la fotosíntesis, presentó diferencias estadísticas entre todos los niveles de estrés y redujo en un $50 \%$ su capacidad fotosintética a los 28 dda. Con respecto a F. tequendamae (figura 2c), se observó una disminución de la fotosíntesis a partir de los 14 dda y hasta los 28 dda con diferencias significativas con valores desde 11 hasta 6 $\mu$ moles $\mathrm{CO}_{2} \mathrm{~m}^{-2} \mathrm{~s}^{-1}$. Con respecto a los arbustos, se pudo observar que hubo una mayor disminución en la tasa fotosintética comparado con los árboles, llegando a valores de $1 \mu \mathrm{mol} \mathrm{CO}_{2} \mathrm{~m}^{-2} \mathrm{~s}^{-1}$ para D. coerulea (figura $2 \mathrm{~d}$ ) y de $4 \mu$ moles $\mathrm{CO}_{2} \mathrm{~m}^{-2} \mathrm{~s}^{-1}$ para $D$. viscosa (figura 2e). S. viarum (figura $2 \mathrm{f}$ ) fue menos afectada, disminuyó su fotosíntesis hasta 7 $\mu$ moles $\mathrm{CO}_{2} \mathrm{~m}^{-2} \mathrm{~s}^{-1}$. Tanto para $D$. coerulea como para $D$. viscosa se observaron diferencias significativas para todos los momentos de estrés, sin embargo, para $S$. viarum solo se presentó diferencias a partir de los 21 dda.

Con respecto a la conductancia estomática (gs), se presentó una disminución para todas las especies evaluadas a medida que el estrés aumenta. Para Q. humboldtii (figura 3a) y F. tequendamae (figura 3c), se presentaron diferencias estadísticas a partir de los 21 dda y los 14 dda respectivamente. Para J. neotropica (figura 3b), D. coerulea (figura $3 \mathrm{~d}$ ), D. viscosa (figura $3 \mathrm{e}$ ) y $S$. viarum (figura $3 \mathrm{f}$ ) se dieron diferencias estadísticas a partir de los 7 dda y en cada uno de los diferentes niveles de estrés por anegamiento. La conductancia estomática disminuyó drásticamente para todas las especies alcanzando valores de alrededor 0.2 mmoles $\mathrm{H}_{2} \mathrm{O}$ $\mathrm{m}^{-2} \mathrm{~s}^{-1}$ en el momento de mayor estrés, excepto para Q. humboldtii, que presentó valores de $0.8 \mathrm{mmo}-$ les $\mathrm{H}_{2} \mathrm{O} \mathrm{m}^{-2} \mathrm{~s}^{-1}$ a los 28 dda.

\section{Fluorescencia de la clorofila a}

La eficiencia máxima del PSII disminuyó significativamente en todas las especies evaluadas a medida que se intensificaba la condición de estrés por anegamiento. Q. humboldtii (figura 4a), 

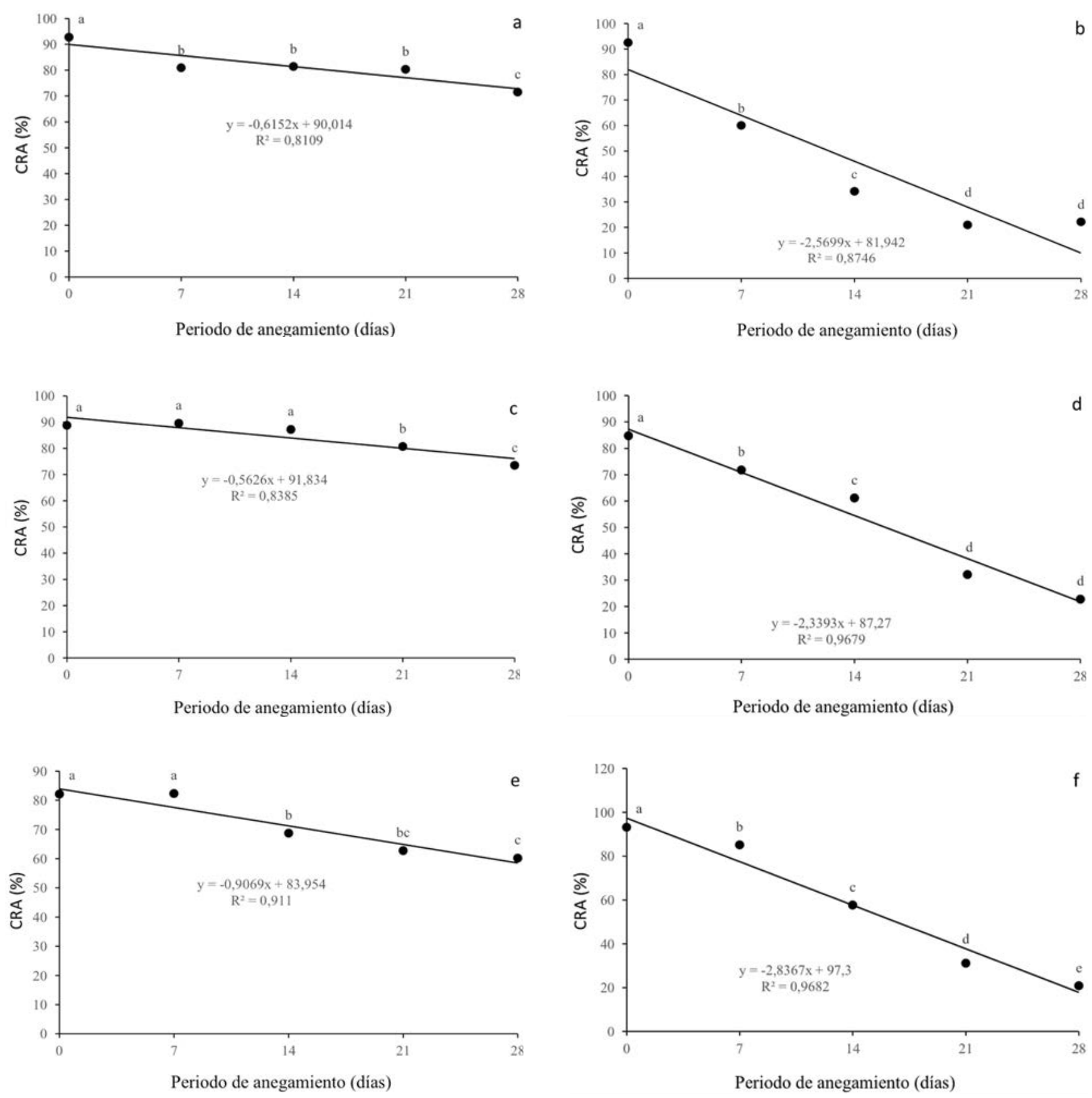

Figura 1. Efecto de diferentes periodos de anegamiento sobre el contenido relativo de agua (CRA) en plantas de (a) Q. humboldtii, (b) J. neotropica, (c) F. tequendamae, (d) D. coerulea, (e) D. viscosa, (f) S. viarum. Promedios seguidos de letras iguales no presentan diferencias estadísticas de acuerdo con la prueba de Tukey (5\%). 

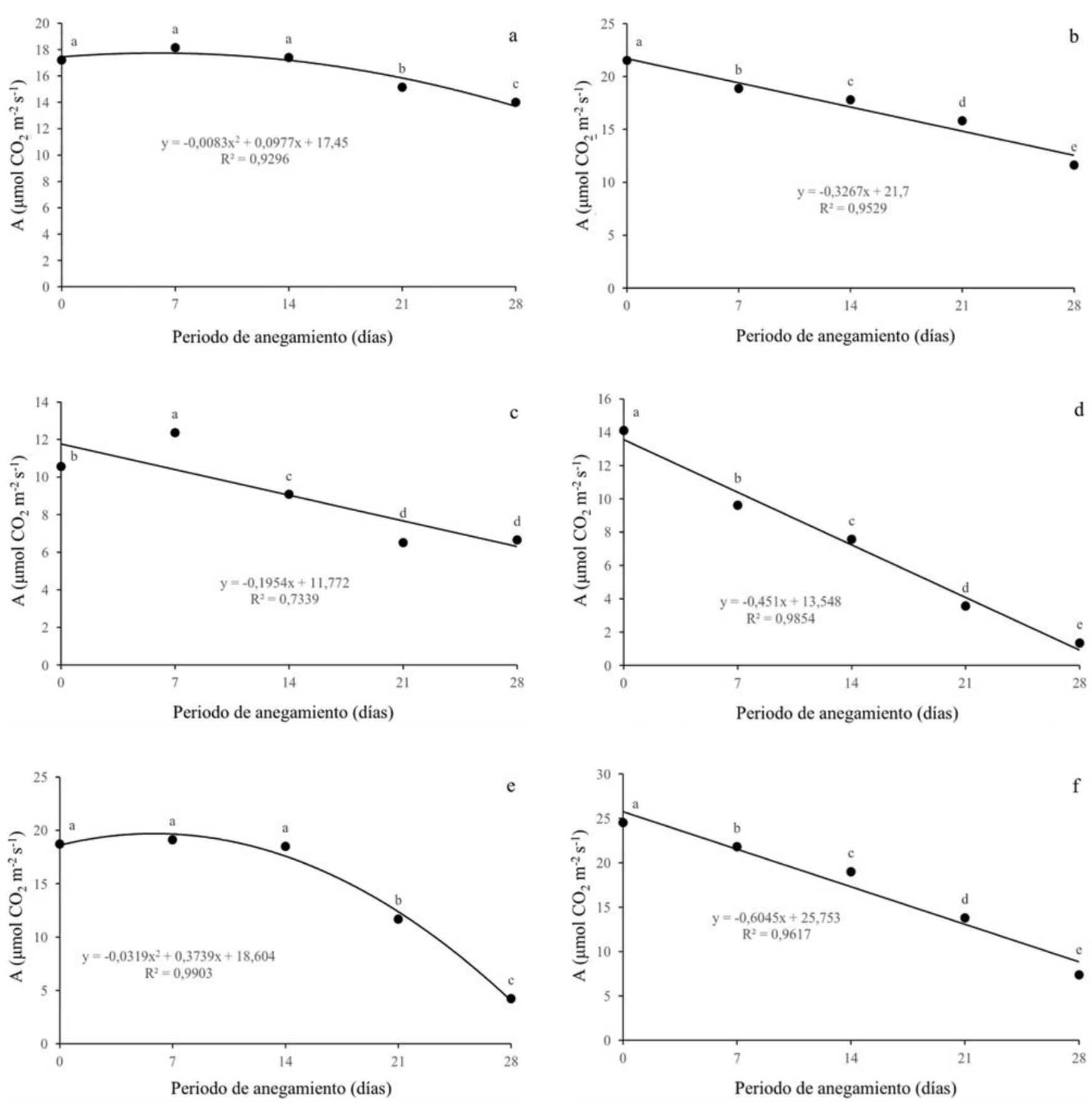

Figura 2. Efecto de diferentes periodos de anegamiento sobre la tasa fotosintética (A) en plantas de (a) Q. humboldtii, (b) J. neotropica, (c) F. tequendamae, (d) D. coerulea, (e) D. viscosa, (f) S. viarum. Promedios seguidos de letras iguales no presentan diferencias estadísticas de acuerdo con la prueba de Tukey $(5 \%)$. 

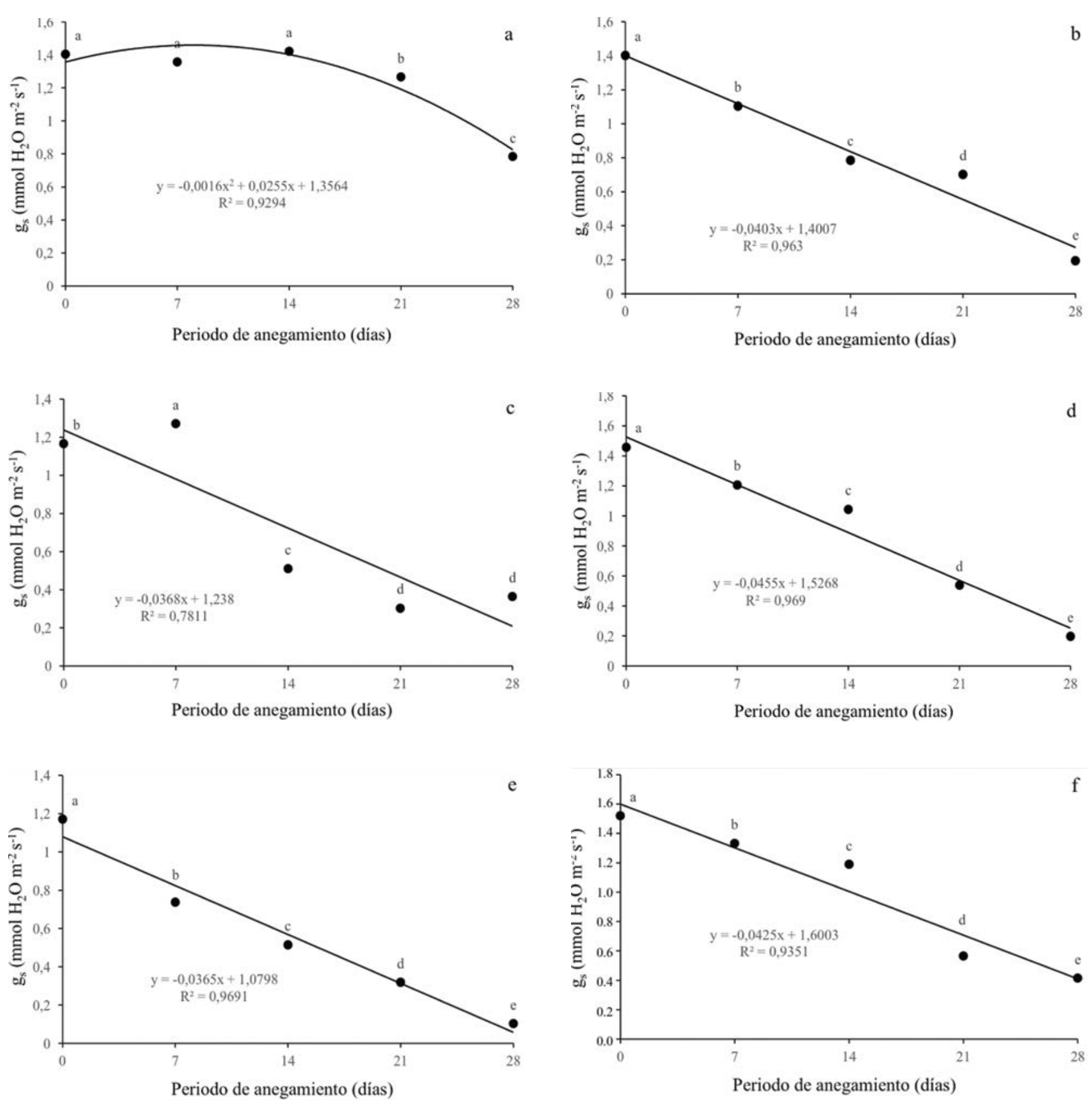

Figura 3. Efecto de diferentes periodos de anegamiento sobre la conductancia estomática (gs) en plantas de (a) $Q$. humboldtii, (b) J. neotropica, (c) F. tequendamae, (d) D. coerulea, (e) D. viscosa, (f) S. viarum. Promedios seguidos de letras iguales no presentan diferencias estadísticas de acuerdo con la prueba de Tukey (5\%). 

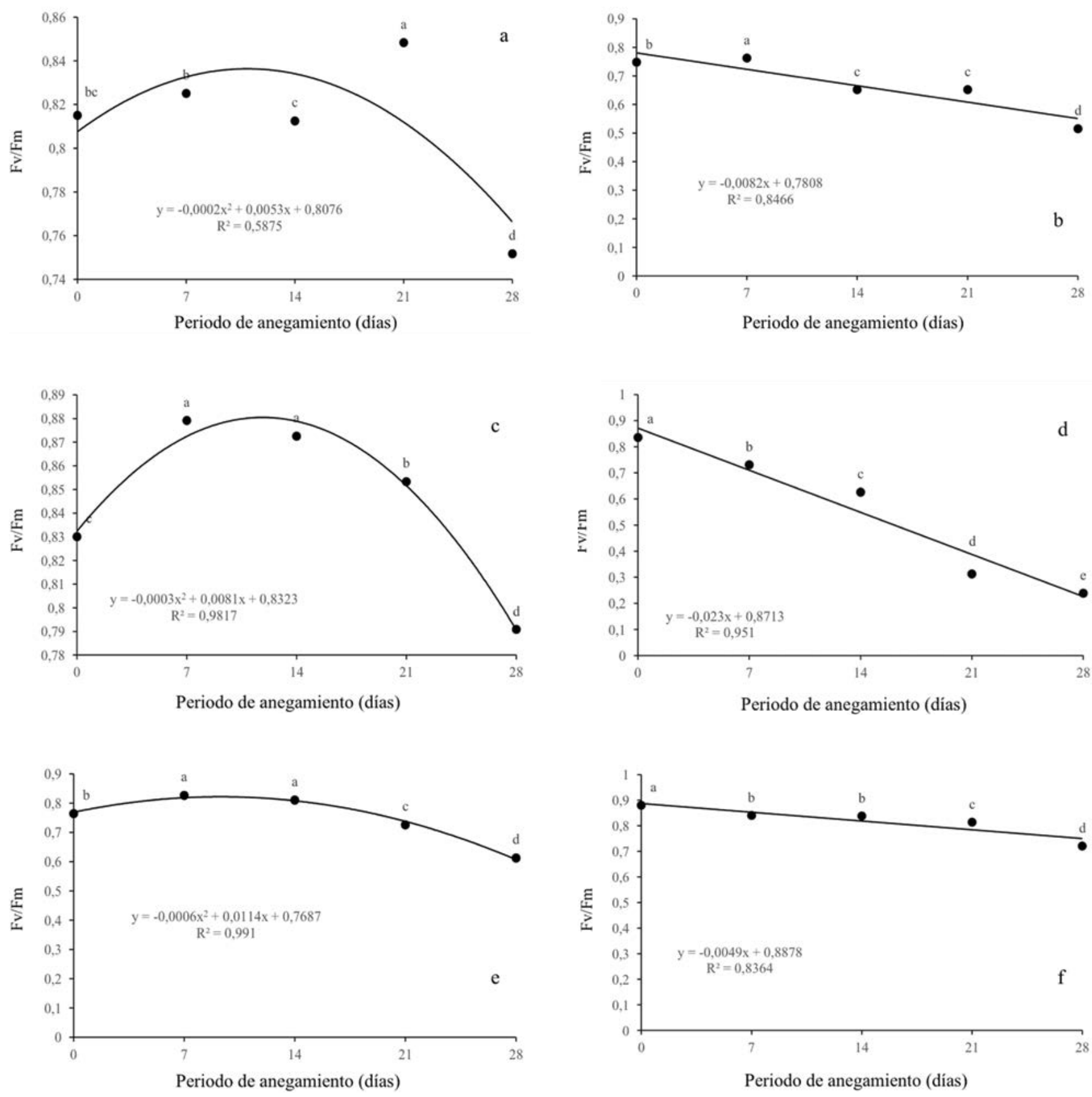

Figura 4. Efecto de diferentes periodos de anegamiento sobre la eficiencia máxima del PSII (Fv/Fm) en plantas de (a) Q. humboldtii, (b) J. neotropica, (c) F. tequendamae, (d) D. coerulea, (e) D. viscosa, (f) S. viarum. Promedios seguidos de letras iguales no presentan diferencias estadísticas de acuerdo con la prueba de Tukey (5\%). 
F. tequendamae (figura 4c) y S. viarum (figura 4f) disminuyeron Fv/Fm por debajo de 0.8 a los 28 dda $(0.75,0.79$ y 0.72 respectivamente). Se evidenció una posible fotoinhibición dinámica. En contraste, J. neotropica (figura 4b) y D. viscosa (figura 4 e) disminuyeron aún más la eficiencia máxima del fotosistema II, con valores de 0.51 y 0.61 para cada uno de ellas, mostraron daños fuertes en el PSII. Por último, la especie que manifestó más daño a nivel del aparato fotosintético fue $D$. coerulea (figura 4d), tuvo diferencias significativas entre todos los tratamientos Ilegando a valores de Fv/Fm de 0.24 a los 28 dda.

\section{Pérdida de electrolitos}

Con respecto a la pérdida de electrolitos se pudo evidenciar que no hubo diferencias estadísticas para Q. humboldtii (figura 5a). Para F. tequendamae (figura $5 \mathrm{c}$ ), se presentó un leve aumento en la PE a partir de los $21 \mathrm{dda}$. Con respecto a $D$. coerulea (figura $5 \mathrm{~d}$ ) se observó un aumento hasta los 14 dda pero la PE se disminuyó tanto a los 21 como a los 28 dda significativamente. Por otro lado, con respecto a J. neotropica (figura 5b), D. viscosa (figura 5e) y $S$. viarum (figura 5f), se presentó un aumento gradual con diferencias significativas entre los diferentes periodos de anegamiento, alcanzando valores superiores al 70 y $90 \%$ cuando las plantas fueron sometidas a 28 dda.

\section{DISCUSIÓN}

El CRA presentó una disminución significativa y severa para J. neotropica (figura 1b), D. coerulea (figura 1d) y $S$. viarum (figura 1f), el anegamiento para estas tres especies afectó el contenido de agua celular de las plantas, debido posiblemente a su incapacidad para retener y absorber las moléculas de agua y generó una pérdida de turgencia celular, lo que limita numerosos procesos en el metabolismo de las plantas (Azhar, Makihara, Naito y Ehara, 2018; Tan et al., 2018; Yan, Zhao, Cui, Han y Wen, 2018). Por otro lado, el contenido relativo de agua disminuyó en una proporción menor para las especies $Q$. humboldtii (figura 1a), F. tequendamae (figura 1c) y D. viscosa (figura 1e), posiblemente debido a los múltiples mecanismos que tienen estas especies para disminuir el potencial hídrico a nivel celular por medio de la síntesis de osmolitos compatibles como prolina, glicina betaína y azúcares solubles, intentando mantener un gradiente de potencial hídrico a favor de la toma de agua (Xiao-Shan y Jian-guo, 2009; MyeongWon y Setsuko, 2015).

Con respecto a los parámetros de intercambio gaseoso, se observó que la primera respuesta que tienen las seis especies a condiciones de anegamiento es el cierre estomático, el cual es gradual y se incrementa ante condiciones de mayor estrés. Para las seis especies se pudo evidenciar que la limitación estomática es la principal causa de la disminución de la fotosíntesis debido a que la planta para evitar la pérdida de agua al interior de sus células y tejidos genera el cierre estomático, lo cual impide, además de la salida de agua, la entrada de $\mathrm{CO}_{2}$, que es fundamental para los procesos fotosintéticos y por lo tanto la planta no tiene suficiente materia prima para la generación de carbohidratos (Rodríguez-Gamir et al., 2011; Duan et al., 2018; Yan et al., 2018); por esta razón, se puede mermar el crecimiento y desarrollo de la misma, al no poder acumular masa seca a través del tiempo (Baracaldo et al., 2014; Nyman y Lindau 2016; Sun, Xu, Zhang, Li y Yan, 2018).

La conductancia estomática se ve afectada por el cierre de estomas, lo que genera menor toma de agua y nutrientes por las plantas y finalmente disminuye la capacidad fotosintética de las mismas (King et al., 2012; Kissmann et al., 2014; Duan et al., 2018). Lo anterior está en concordancia con lo que afirman Anderson y Pezeshki (2001), que evidenciaron en dos especies de Quercus que bajo 

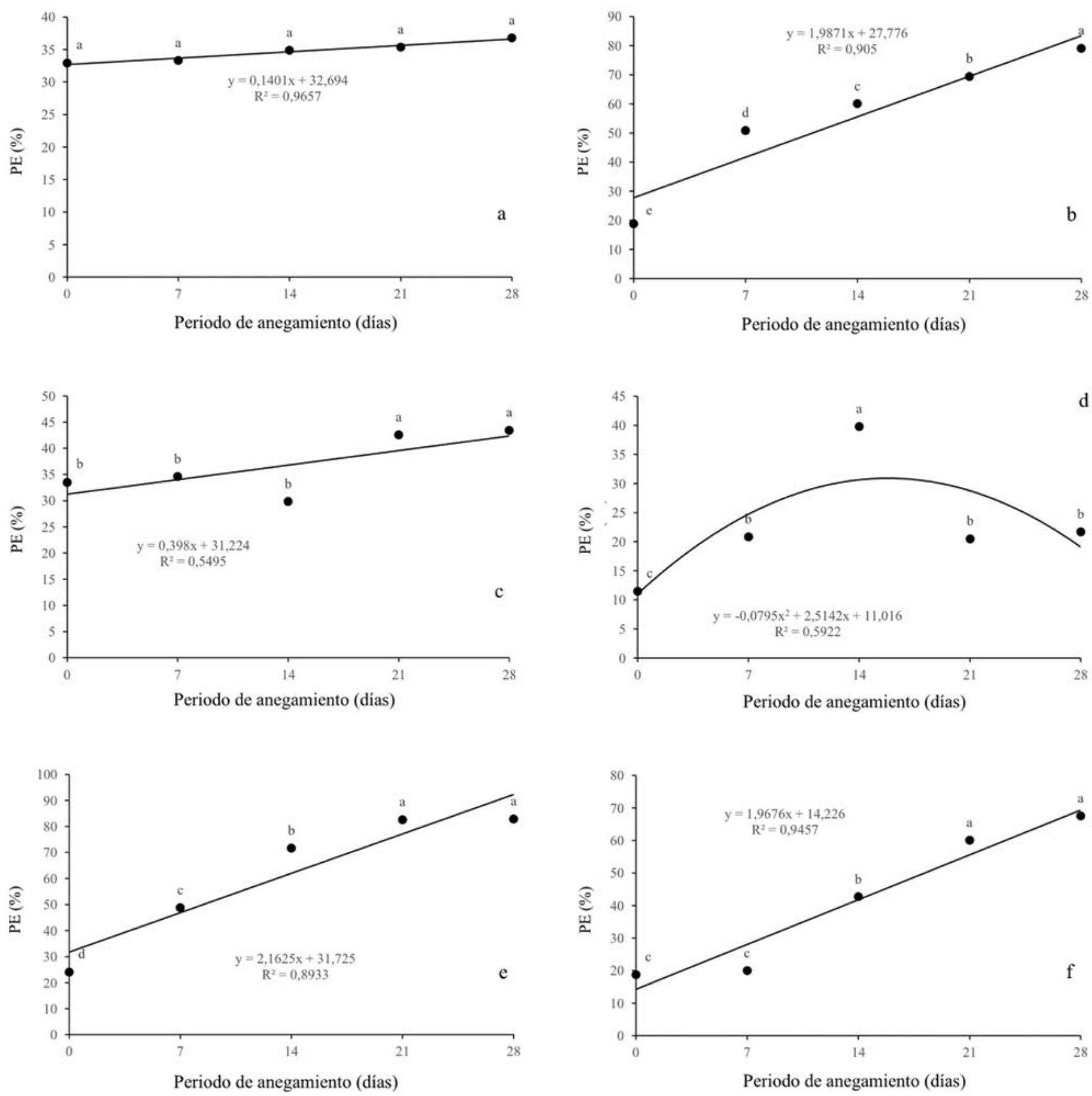

Figura 5. Efecto de diferentes periodos de anegamiento sobre la pérdida de electrolitos en plantas de (a) Q. humboldtii, (b) J. neotropica, (c) F. tequendamae, (d) D. coerulea, (e) D. viscosa, (f) S. viarum. Promedios seguidos de letras iguales no presentan diferencias estadísticas de acuerdo con la prueba de Tukey $(5 \%)$. 
condiciones de anegamiento se disminuye la conductancia estomática y por ende la concentración de $\mathrm{CO}_{2}$ interno, esto afecta la tasa fotosintética en más de un 70\%. También con los resultados mostrados por Corcobado et al. (2014), que demuestran que bajo condiciones de anegamiento la tasa fotosintética se puede reducir en más de un 50\% en plantas de Quercus ilex, debido tanto a efectos de la limitación estomática como a efectos de la limitación no estomática, que genera acumulación de especies reactivas de oxígeno y, por ende, daño a nivel de membranas y fotosistemas (Benedetti, Ferreira y Fernandes, 2015; Sun et al., 2018).

La eficiencia máxima del PSII se vio realmente afectada por las condiciones de anegamiento, principalmente para $D$. coerulea (figura $4 d$ ). que disminuyó drásticamente evidenciando graves daños a nivel del PSII, posiblemente por per-oxidación de la proteína D1 y que seguramente no puede ser sintetizada a la misma o a mayor velocidad comparado con la degradación a la que está siendo sometida por el nivel de especies reactivas de oxígeno en esta especie (Ohnishi et al., 2005; Nishiyama, Allakhverdiev y Murata, 2006; Andrade, Dázio, Santos, Silva y Donizeti, 2018).

Los fotosistemas también se pueden ver afectados por la peroxidación lipídica de las membranas celulares afectando los procesos fotosintéticos y la respuesta de esta especie a condiciones de estrés. J. neotropica (figura 4b) y D. viscosa (figura 4e) redujeron en menor medida la eficiencia máxima del fotosistema II, con valores de 0.51 y 0.61 respectivamente, evidenciando que para $28 \mathrm{dda}$ existen daños en el fotosistema que se pueden recuperar si la condición estresante se retira. Para las demás especies se observó una fotoinhibición dinámica, en la cual se evidenció una disminución de este parámetro por encima de 0.7, lo que indica que los fotosistemas rápidamente se están recuperando frente a las condiciones de estrés por una alta tasa de recambio en la proteína D1 del fotosistema II, como ha sido observado en otras investigaciones bajo condiciones de anegamiento (Pietrini, Chaudhuri, Thapliyal y Massacci, 2005; Guidi y Calatayud, 2014; Benedetti et al., 2015; Duan et al., 2018; Yan et al., 2018).

Por último, el aumento en el porcentaje de electrolitos sugiere un daño en las membranas cambiando su permeabilidad (González, Delgado, Zabaleta y Herrera, 2009). Este daño pudo ser causado por la pérdida de la capa de hidratación de la membrana o por la peroxidación de los lípidos causada por el aumento de ROS (Zhao et al., 2000; Simova-Stoilova, Demirevska, Petrova, Tsenov y Feller, 2009; Andrade et al., 2018), lo que permite mayor permeabilidad en sus membranas y, por consiguiente, mayor pérdida de electrolitos (González et al., 2009). En la figura 5 se puede observar que para la especie Q. humboldtii (figura 5a) no se presentó pérdida de electrolitos significativa para ningún momento de anegamiento, lo que significa que esta especie tolera muy bien la condición de estrés ya sea por una baja producción de especies reactivas de oxígeno (ROS) a nivel celular o una alta eficiencia en el control de estas ROS por medio de la síntesis y actividad de antioxidantes enzimáticos y no enzimáticos (Devkota y Kumar, 2010). Una tendencia similar se obtuvo en $F$. tequendamae (figura 5c), la cual no presentó un aumento significativo de pérdida de electrolitos sino hasta los $21 \mathrm{dda}$, los cuales permanecieron constantes a los 28 dda: una respuesta similar a $Q$. humboldtii.

Ahora bien, J. neotropica (figura 5b), D. viscosa (figura 5e) y S. viarum (figura 5f) presentaron un aumento gradual con diferencias significativas entre los diferentes periodos de anegamiento, alcanzando valores superiores al 70 y $90 \%$. Esto señala que, aunque las plantas pueden tener algún mecanismo para impedir el daño a nivel de membranas celulares, este no es suficiente y si el factor estresante no es retirado posterior a los 28 dda posiblemente se genere un daño irreversible a nivel de las estructuras celulares. 


\section{CONCLUSIONES}

De acuerdo a la respuesta fisiológica obtenida por medio de los diferentes parámetros evaluados se puede concluir que tanto $Q$. humboldtii como $F$. tequendamae, tienen una alta tolerancia a condiciones de anegamiento, debido a que no varía su contenido relativo de agua, no se afecta el estado del fotosistema II y no hay daño a nivel de membranas.

Especies como J. neotropica, D. viscosa y S. viarum, se pueden recomendar para zonas con altas precipitaciones, sin que el anegamiento sea una constante en la zona. Pese a que su recuperación tiende a ser rápida, tanto a nivel de fotosíntesis, conductancia estomática y estado del aparato fotosintético, las plantas de estas tres especies arbóreas presentaron el mayor daño a nivel de las membranas, seguramente por la mayor acumulación de especies reactivas de oxígeno y la menor actividad de enzimas antioxidantes.

No se recomienda el uso de la especie $D$. coerulea en zonas con altas precipitaciones y que tiendan a inundarse, debido a que, según el estudio, solo toleran 15 días de anegamiento y decrecen drásticamente parámetros como Fv/Fm. El anegamiento daña los fotosistemas y su recuperación es muy lenta. De acuerdo al estado actual del arbolado urbano de Bogotá, la especie $D$. coerulea es la que presenta menor número de individuos en la ciudad, con respecto a las otras especies evaluadas. Por este motivo, se sugiere que su establecimiento solo se realice en zonas que no sean inundables $y$, en lo posible, que no presenten un exceso en la precipitación.

Como se puede observar, las especies tolerantes a condiciones de anegamiento presentan un comportamiento similar con respecto a la protección de estructuras celulares como las membranas (pérdida de electrolitos) y los fotosistemas (Fv/Fm). Así pues, estos parámetros fisiológicos pueden ser usados como marcadores de tolerancia a condiciones de anegamiento en especies arbóreas.

\section{CONFLICTO DE INTERESES}

Los autores declaran no tener conflicto de intereses.

\section{CONTRIBUCIÓN POR AUTOR}

Los autores son los únicos responsables de la obra en todos los aspectos que condujeron a la elaboración de su publicación.

\section{REFERENCIAS}

Aldana, F., García, P. y Fischer, G. (2014). Effect of waterlogging stress on the growth, development and symptomatology of cape gooseberry (Physalis peruviana L.) Plants. Revista de la Academia Colombiana de Ciencias Exactas, 38(149), 393-400. DOI: https://doi.org/10.18257/raccefyn.114

Ali, H., Kabir, N., Muhammad, A., Raza, M., Ghulam, S., Iqbal, N. y Nadeem, S. (2014). Hautriwaic acid as one of the hepatoprovec tive constituent of Dodonaea viscosa. Phytomedicine, 21, 131-140. DOI: https://doi.org/10.1016/j.phymed.2013.08.019

Almeida, T., Pinto, G., Correia, B., Santos, C. y Concalves, S. (2013). QsMYB1 expression is modulated in response to heat and drought stresses and during plant recovery in Quercus suber. Plant Physiology and Biochemistry, 73, 274-281. DOI: https://doi.org/10.1016/j.plaphy.2013.10.007

Anderson, P. y Pezeshki, R. (2001). Effects of flood pre-conditioning on responses of three bottomland tree species to soil waterlogging. Journal of Plant Physiology, 158, 227-233. DOI: https://doi.org/10.1078/0176-1617-00267

Andrade, C., Dázio, K., Santos, M., Silva, D. y Donizeti, J. (2018). Hydrogen peroxide promotes the tolerance of soybeans to waterlogging. Scientia Horticulturae, 232, 40-45. DOI: https://doi.org/10.1016/j. scienta.2017.12.048

Azhar, A., Makihara, D., Naito, H. y Ehara, H. (2018). Evaluating sago palm (Metroxylon sagu Rottb.) 
photosynthetic performance in waterlogged conditions: utilizing pulse-amplitude-modulated (PAM) fluorometry as a waterlogging stress indicator. Journal of the Saudi Society of Agricultural Sciences. DOI: https://doi.org/10.1016/j.jssas.2018.05.004

Bailey-Serres, J. y Voesenek, L. (2008). Flooding Stress: Aclimation and Genetic Diversity. Annual Review of Plant Biology, 59, 313-39. DOl: https://doi. org/10.1146/annurev.arplant.59.032607.092752

Banach, K., Banach, A., Lamers, L., Kroon, H., Bennicelli, R., Smits, A. y Visser, J. (2009). Differences in flooding tolerance between species from two weland habitats with contrasting hydrology: implications for vegetation development in future flodwater retention areas. Annals of Botany, 103, 341-351. DOI: https://doi.org/10.1093/aob/mcn183

Baracaldo, A., Carvajal, R., Romero, A., Prieto, A., García, F., Fischer, G. y Miranda, D. (2014). El anegamiento afecta el crecimiento y producción de biomasa en tomate chonto (Solanum lycopersicum L.) cultivado bajo sombrío. Revista Colombiana de Ciencias Hortícolas, 8(1), 92-102. DOI: https://doi.org/10.17584/rcch.2014v8i1.2803

Benedetti, L., Ferreira, G. y Fernandes, S. (2015). Chlorophyll a fluorescence in Annona emarginata (Schltdl.) H. Rainer plants subjectd to wáter stress and after rehydration. Scientia Horticulturae, 184, 23-30. DOI: https://doi.org/10.1016/j. scienta.2014.12.019

Bhatt, R., Upreti, K., Divya, M., Bath, S., Pavithra, C. y Sadashiva, A. (2015). Interspecific grafting to enhance physiological resilience to floodingstress in tomato (Solanum lycopersicum L.). Scientia Horticulturae, 182, 8-17. DOI: https://doi.org/10.1016/j. scienta.2014.10.043

Borowski, J. y Pstragowska, M. (2010). Effect of street conditions, including saline aerosol, on growht of the Small-leaved limes. Rocznik Polskiego Towarzystwa Dendrologicznegro, 58, 15-24

Campoe, O., Ianelli, C., Stape, J., Cook, R., Mendes, J. y Vivian, R. (2014). Atlantic forest tree species responses to silvicultural practices in a degraded pasture restoration plantation: From leaf physiology to survival and initial growth. Forest Ecology and
Management, 313, 233-242. DOI: https://doi.org/10.1016/j.foreco.2013.11.016

Caudle, K. y Maricle, B. (2014). Physiologycal relationship between oil tolerance and flooding tolerance in marsh plants. Environmental and Experimental Botany, 107, 7-14. DOI: https://doi.org/10.1016/j. envexpbot.2014.05.003

Cekstere, G., Nikodemus, O. y Osvalde, A. (2008). Toxic impact of the de-icing material to street greenery in Riga, Latvia. Urban Forestry \& Urban Greening, 7, 207-217. DOI: https://doi.org/10.1016/j. ufug.2008.02.004

Colmer, T., Cox, M. y Voesenek, L. (2006). Root aeration in rice (Oryza sativa): evaluation of oxygen, carbon dioxide and ethylene as possible regulators of root acclimatizations. New Phytologist, 170, 767-778. DOI: https://doi. org/10.1111/j.1469-8137.2006.01725.x

Corcobado, T., Cubera, E., Juárez, E., Moreno, G. y SoIla, A. (2014). Drought events determine performance of Quercus ilex seedlings and increase their suspectibility to Phytophthora cinnamomi. Agricultural and Forest Meteorology, 192-193, 1-8. DOI: https://doi.org/10.1016/j.agrformet.2014.02.007

Dat, J., Capelli, N, Folzer., H, Bourgeade, P. y Badot, P. (2004). Sensing and signalling during plant flooding. Plant Physiology and Biochemistry, 42, 273-282. DOI: https://doi.org/10.1016/j.plaphy.2004.02.003

Dell’Amico, J., Torrecillas, A., Rodríguez, P., Morales, D. y Sánchez-Blanco, M. (2001). Differences in the effects of flooding the soil early and late in the photoperiod on the water relations of pot-grown tomato plants. Plant Science, 160, 481-487. DOI: https://doi.org/10.1016/S0168-9452(00)00409-X

Devkota, A. y Kumar, P. (2010). Effects of different light levels on the growht traits and yield of Centella asiática. Journal of Scientific Research, 5, 226-230.

Du, k., Xu, L., Wu, H., Tu, B. y Zheng, B. (2012). Ecophysiological and morphological adaption to soil flooding of two poplar clones differing in flood-tolerance. Flora, 207, 96-106. DOI: https://doi.org/10.1016/j.flora.2011.11.002

Duan, H., Ma, Y., Liu, R., Li, Q., Yang, Y. y Song, J. (2018). Effect of combined waterlogging and 
salinity stresses on euhalophyte Suaeda glauca. Plant Physiology and Biochemistry, 127, 231-237. DOI: https://doi.org/10.1016/j.plaphy.2018.03.030

Glenz, C., Lougulescu, I., Kienast, F. y Schalaepfer, R. (2008). Modelling the impact of flooding stress on the growth performance of woody species using fuzzy logic. Ecological Modelling, 218, 18-28. DOI: https://doi.org/10.1016/j.ecolmodel.2008.06.008

González, R., Delgado. A., Zabaleta, H. y Herrera, E. (2009). Cytokinin BAP promotes the accumulation of hexoses and increases the activity of phosphoenolpyruvate carboxylase and phosphoenolpturvate carboxikinase during the delay of leaf senescence in wheat. Agrociencia, 43, 379-391.

Guidi, L. y Calatayud, A. (2014). Non-invasive tolos to estimate stress-induced changes in photosynthetic permormance in plants inhabiting Mediterranean areas. Environmental and Experimental Botany, 103, 42-52. DOI: https://doi.org/10.1016/j. envexpbot.2013.12.007

Hill, K., Guerin, G., Hill, R. y Watling, J. (2014). Temperature influences stomatal density and maximum potential water loss through stomata of Dodonaea viscosa subsp. Angustissima along a latitude gradient in southern Australia. Australian Journal of Botany, 62, 657-665. DOI: https://doi.org/10.1071/ BT14204

Hundecha, Y. y Bárdossy, A. (2004). Modeling of the effect of land use changes on the runoff generation of a river basin through parameter regionalization of a watershed model. Journal of Hydrology, 292, 281-295. DOI: https://doi.org/10.1016/j.jhydrol.2004.01.002

Hussain, J., Rehman, N., Al-Harrasi, A., Ali, L., Latif, A. y Abdullah, M. (2013). Essential oil composition and nutrient analysis of selected medicinal plants in sultanate of Oman. Asian Pacific Journal of Tropical Disease, 3(6), 421-428. DOI: https://doi. org/10.1016/S2222-1808(13)60095-X

Jim, C. y Zhang, H. (2013). Species diversity and spatial differentiation of old-valuable trees in urban Hong Kong. Urban Forestry \& Urban Greening, 12, 171-182. DOI: https://doi.org/10.1016/j. ufug.2013.02.001
Kamdem, H., Chabert, P., Tane, P., Noté, O., Feussi, M., Peluso, J., Muller, C., Kikuchi, H., Oshima, Y. y Lobstein, A. (2012). Labdane-type diterpenes and flavones from Dodonaea viscosa. Fitoterapia, 83, 859-863. DOI: https://doi.org/10.1016/j. fitote.2012.03.014

King, C., Robinson, J. y Cameron, R. (2012). Flooding tolerance in four "Garrigue" landscape plants: Implications for their future use in the urban landscapes of north-west Europe? Landscape and Urban Planning, 107, 100-110. DOI: https://doi.org/10.1016/j. landurbplan.2012.05.005

Kirkpatrick, J., Davison, A. y Daniels, G. (2013). Sinners, scapegoats or fashion victims? Understanding the deaths of trees in the green city. Geoforum, 48, 165-176. DOI: https://doi.org/10.1016/j. geoforum.2013.04.018

Kissmann, C., Veiga, E., Eichemberg, M. y Habermann, G. (2014). Morphological effects of flooding on Styrax pohlii and the dynamics of physiological responses during flooding and post-flooding conditions. Aquatic Botany, 119, 7-14. DOI: https://doi. org/10.1016/j.aquabot.2014.06.007

Kreuzwieser, J., Hauberg, J., Howell, K., Carroll, A., Rennenberg, H., Havery, A. y Whelan, J. (2009). Differential response of gray poplar leaves and roots underpins stress adaptation during hipoxia. Plant Physiology, 149, 461-473. DOI: https://doi. org/10.1104/pp.108.125989

Liu, F., Shahanzari, A. y Andersen, M. (2005). ABA regulated stomatal control and photosynthetic water use efficiency of potato (Solanum tuberosum L.) during progressive soil drying. Plant Science, 168, 831-836. DOI: https://doi.org/10.1016/j. plantsci.2004.10.016

Lucassen, T., Bobbink, R., Smolders, A., Van der Ven, M., Lamers, M. y Roelofs, J. (2002). Interactive effects of low $\mathrm{pH}$ and high ammonium levels responsible for the decline of Cirsium dissectum (L.) Hill. Plant Ecology, 165, 45-52. DOI: https://doi.org/10.1023/A:1021467320647

McPherson, E., Berry, A. y Svan, N. (2018). Performance testing to identify climate-ready trees. 
Urban Forestry \& Urban Greening, 29, 28-39. DOI: https://doi.org/10.1016/j.ufug.2017.09.003

Moreno, A. y Fischer, G. (2014). Efectos del anegamiento en los frutales. Una revisión. Temas agrarios, 19(1), 106-23. DOI: https://doi.org/10.21897/ rta.v19i1.729

Mostafa, A., El-Hela, A., Mohammad, A., Jacob, M., Cutler, S. y Ross, S. (2014). New secondary metabolites from Dodonaea viscosa. Phytochemistry Letters, 8, 10-15. DOI: https://doi.org/10.1016/j. phytol.2013.12.008

MyeongWon, O. y Setsuko, K. (2015). Characterization of proteins in soybean roots under flooding and drought stresses. Journal of Proteomics, 114, 161-181. DOI: https://doi.org/10.1016/j. jprot.2014.11.008

Nishiyama, Y., Allakhverdiev, I. y Murata, N. (2006). A new paradigm for the action of reactive oxygen species in the photoinhibition of photosystem II. Biochimica et Biophysica Acta, 1757, 742-749. DOI: https://doi.org/10.1016/j.bbabio.2006.05.013

Nyman, J. y Lindau, C. (2016). Nutrient availability and flooding stress interact to affect growth and mercury concentration in Taxodium distichum (L.) Rich. seedlings. Environmental and Experimental Botany, 125, 77-86. DOI: https://doi.org/10.1016/j. envexpbot.2016.02.004

Ohnishi, N., Allakhverdiev, S.I., Takahashi, S., Higashi, S., Watanabe, M., Nishiyama, Y.y Murata, N. (2005). Two-step mechanism of photodamage to photosystem II: step 1 occurs at the oxygen-evolving complex and step 2 occurs at the photochemical reaction center. Biochemistry, 44, 8494-8499. DOI: https://doi.org/10.1021/bi047518q

Ordoñez, C. (2015). Adopting public values and climate change adaptation strategies in urban forest management: A reviewand analysis of the relevant literature. Journal of Environmental Management, 164(1), 215-221.

Pardos, J. A. (2010). Respuestas de las plantas al anegamiento del suelo. Investigación agraria. Sistemas y recursos forestales, 13(1), 101-107.

Paz, G. (2012). Genetic variability of common oak (Quercus humboldtii Bonpl.) in the "Macizo colombiano" region. Biotecnología en el sector Agropecuario y Agroindustrial, 10(2), 110-116.

Pérez, C., Villalba, J. y Almanza, M. (2013). Phenology oak (Quercus humboldtii Bonpl.) in Popayán (Cauca, Colombia). Biotecnología en el Sector Agropecuario y Agroindustrial, 2, 145-154.

Pietrini, F., Chaudhuri, D., Thapliyal, P. y Massacci, A. (2005). Analysis of chlorophyll fluorescence transients in mandarin leaves during a photo-oxidative cold shock and recovery. Agriculture, Ecosystem and Environment, 106, 189-198. DOI: https://doi.org/10.1016/j.agee.2004.10.007

Quintero, O. y Jaramillo, S. (2012). Germination and rescue in vitro of immature embryos of black cedar (Juglans neotropica Diels). Acta Agronómica, 61(1), 52-60.

Rodríguez, P., Torrecillas, A., Morales, M., Ortuño, M. y Sánchez, M. (2005). Effects of $\mathrm{NaCl}$ salinity and water stress on growth and leaf water relations of Asteriscus maritimus plants. Environmental and Experimental Botany, 53, 113-123. DOI: https://doi. org/10.1016/j.envexpbot.2004.03.005

Rodríguez-Gamir, J., Ancillo, G., González, M., Millo, E., Iglesias, D. y Giner, A. (2011). Root signaling and modulation of stomatal closure in flooded citrus seedlings. Plant Physiology and Biochemistry, 49, 636-645. DOI: https://doi.org/10.1016/j. plaphy.2011.03.003

Rowshan, V., Farhadi, F. y Najafian, S. (2014). The essential oil of Dodonaea viscosa leaves is allelopathic to rosemary (Rosmarinus officinalis L.). Industrial Crops and Products, 56, 241-245. DOI: https://doi. org/10.1016/j.indcrop.2014.03.011

Salazar, S., Francés, F., Blume, T., Francke, T., Bronstert, A. y Blóschl, G. (2012). A comparative analysis of the effectiveness of flood management measures based on the concept of "retaining water in the landscape" in different European hydro-climatic regions. Natural Hazards and Earth System Sciences, 12, 3287-3306. DOI: https://doi.org/10.5194/ nhess-12-3287-2012

Sepulveda, Y., Diez, M., Moreno, F., León, J. y Osorio, N. (2014). Effects of light intensity and fertilization on the growth of Andean oak seedlings at 
nursery. Acta Biológica Colombiana, 19(2), 211-220.

DOI: https://doi.org/10.15446/abc.v19n2.40091

Sghaier-Hammami, B., Valero-Galván, J., Romero-Rodríguez, C, Navarro-Cerrillo, R., Abdelly, C. y Jorrín-Novo, J. (2013). Physiological and proteomics analyses of Holm oak (Quercus ilex subsp. ballota (Desf.) Samp.) responses to Phytophthora cinnamomi. Plant Physiology and Biochemistry, 71, 191-202. DOI: https://doi.org/10.1016/j. plaphy.2013.06.030

Simova-Stoilova, L., Demirevska, K., Petrova, T., Tsenov, N. y Feller, U. (2009). Antioxidative protection and proteolytic activity in tolerant and sensitive wheat (Triticum aestivum L.) varieties subjected to long-term field drought. Plant Growth Regulation, 58, 107-117. DOI: https://doi.org/10.1007/ s10725-008-9356-6

Sistema de Información para la Gestión del Arbolado Urbano (Sigau) (2018). Sistema de Información para la Gestión del Arbolado Urbano. Recuperado de DOI: http://www.jbb.gov.co/index.php/sigau

Sjoman, H. y Nielsen, A. (2010). Selecting trees for urban paved sites in Scandinavia- A review of information on stress tolerance and its relation to the requirements of tree planners. Urban Forestry \& Urban Greening, 9, 281-293. DOI: https://doi.org/10.1016/j.ufug.2010.04.001

Sun, X., Xu, Y., Zhang, Q., Li, X. y Yan, Z. (2018). Combined effect of water inundation and heavy metal son the photosynthesis and physiology of Spartina alterniflora. Ecotoxicology and Environmental Safety, 153, 248-258. DOI: https://doi.org/10.1016/j. ecoenv.2018.02.010

Swoczyna, T., Klaji, H., Pietkiewicz, S. y Borowsky, J. (2015). Ability of various tree species to acclimation in urban environments probed with the JIP-test. Urban Forestry and Urban Greening, 14, 544-533. DOI: https://doi.org/10.1016/j.ufug.2015.05.005

Tan, X., Xu, H., Khan, S., Equiza, M., Lee, S., Vaziriyeganeh, M. y Zwiazed, J. (2018). Plant water transport and aquaporins in oxygen-deprived environments. Journal of Plant Physiology, 227. DOI: DOI: http://doi.org/10.1016/j.jplph.2018.05.003

Tullah, B., Hussain, F. y Ibrar, M. (2010). Allelopathic potential of Dodonaea Viscosa (L.) Jacq. Pakistan Journal of Botany, 42(4), 2383-2390.

Voesenek, L. y Serres, J. (2013). Flooding tolerance: $\mathrm{O}_{2}$ sensing and survival strategies. $\mathrm{Cu}$ rrent Opinion in Plant Biology, 16(5), 647-653. DOI: https://doi.org/10.1016/j.pbi.2013.06.008

Wilby, R. (2008). Constructing climate change scenarios of urban heat island intensity and air quality. Environment and Planning B. Planning and Design, 35, 902-919. DOI: https://doi.org/10.1068/ b33066t

Xiao-Shan, W. y Jian-Guo, H. (2009). Changes of proline content, activity and active isoforms of antioxidative enzymes in two alfalfa cultivars under salt stress. Agricultural Sciences in China, 8(4), 431-440. DOI: https://doi.org/10.1016/S1671-2927(08)60229-1

Yan, K., Zhao, S., Cui, M., Han, G. y Wen, P. (2018). Vulnerability of photosynthesis and photosystem I in Jerusalem artihoke (Helianthus tuberosus L.) exposed to waterlogging. Plant Physiology and Biochemistry, 125, 239-246. DOI: https://doi.org/10.1016/j.plaphy.2018.02.017

Yetisir, H., Caliskan, M., Soylu, S. y Sakar, M. (2006). Some physiological and growth responses of watermelon (Citrullus lanatus Thunb-Matsum. and Nakai) grafied onto Lagenaria siceraria to flooding. Environmental and Experimental Botany, 58, 1-8. DOI: https://doi.org/10.1016/j. envexpbot.2005.06.010

Zhao, H., Wang, B., Liu, Y., Duan, D. Cai, S. y Sakanishi, A. (2000). Influence of water stress on the lipid physical state of plasma membranes from P. betuloefolia. Bqe leaves. Colloids and Surfaces B: Biointerfaces, 19, 181-185. DOI: https://doi. org/10.1016/S0927-7765(00)00153-3

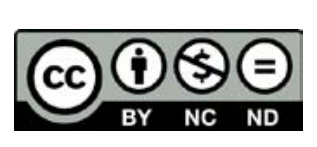

Colombia Forestal •ISSN 0120-0739 • e-ISSN 2256-201X • Bogotá-Colombia • Vol. 22 No. 1. Enero-Junio 2019 • pp. 51-67. [67] 